\title{
A better approximation ratio for the Vertex Cover problem
}

\author{
George Karakostas \\ Dept. of Computing and Software \\ McMaster University
}

October 5, 2004

\begin{abstract}
We reduce the approximation factor for Vertex Cover to $2-\Theta\left(\frac{1}{\sqrt{\log n}}\right)$ (instead of the previous $2-\Theta\left(\frac{\log \log n}{\log n}\right)$, obtained by Bar-Yehuda and Even [2], and by Monien and Speckenmeyer [10]). The improvement of the vanishing factor comes as an application of the recent results of Arora, Rao, and Vazirani [1] that improved the approximation factor of the sparsest cut and balanced cut problems. In particular, we use the existence of two big and well-separated sets of nodes in the solution of the semidefinite relaxation for balanced cut, proven in [1]. We observe that a solution of the semidefinite relaxation for vertex cover, when strengthened with the triangle inequalities, can be transformed into a solution of a balanced cut problem, and therefore the existence of big well-separated sets in the sense of [1] translates into the existence of a big independent set.
\end{abstract}

\section{Introduction}

One of the most well-studied problems in combinatorial optimization is the vertex cover (VC) problem: given a graph $G=(V, E)$, we look for a minimum size subset of vertices such that for every $(u, v) \in E$, at least one of $u, v$ belongs to this subset. In the weighted version of $\mathrm{VC}$, each vertex has an integral weight, and we are looking for the minimum total weight subset of vertices with the property above.

Since the complexity of VC has been heavily studied since Karp's original proof of its NPcompleteness [8], the related bibliography is vast and cannot be covered, of course, in this introductory note. We mention here that VC is known to be APX-complete [11], and moreover it cannot be approximated within a factor of 1.36 [5], unless $\mathrm{P}=\mathrm{NP}$. A 2-approximation on the other hand can be trivially obtained by taking all the vertices of a maximal matching in the graph.

Improving this simple 2-approximation algorithm has been a quite non-trivila task. The best approximation algorithms known before this work were published 20 years ago by Bar-Yehuda and Even [2], and by Monien and Speckenmeyer [10]. They achieved an approximation factor of $2-\frac{\ln \ln n}{2 \ln n}$, where $n$ is the number of vertices. If $\Delta$ is the maximum degree of the graph, Halperin [7] showed that a factor of $2-(1-o(1)) \frac{2 \ln \ln \Delta}{\ln \Delta}$ can be achieved by using the semidefinite programming (SPD) relaxation of $\mathrm{VC}$.

In this work we use a stronger SDP relaxation to improve the approximation factor achieved in polynomial time to $2-\Theta\left(\frac{1}{\sqrt{\log n}}\right)$. We observe that the introduction of all the so-called triangle inequalities to the standard SDP relaxation of $\mathrm{VC}$ is, in fact, very similar to the balanced cut SDP relaxation used by Arora, Rao, and Vazirani [1]. Then we use one of the main results of [1], which asserts that in the solution of this SDP, there are two big and well-separated vertex subsets. At the same time, we show that edges that were not covered by a trivial initial rounding are too big to have both of their endpoints in either of these two sets. Hence, one of these two big subsets has to be a 
big independent set, which can be excluded. We show this process first for the unweighted VC, and then we show how it can be extended to the weighted case in a straight-forward manner. We end with some open problems.

\section{The unweighted case}

The following is a semidefinite-programming relaxation of unweighted Vertex Cover (VC) for a graph $G=(V, E)$ with $n$ nodes:

$$
\begin{array}{rrr}
\min \sum_{i=1}^{n} \frac{1+v_{0} v_{i}}{2} \text { s.t. } & \\
\left(v_{0}-v_{i}\right)\left(v_{0}-v_{j}\right) & =0, & \forall(i, j) \in E \\
\left(v_{i}-v_{j}\right)\left(v_{i}-v_{k}\right) \geq 0, & \forall i, j, k \in V \cup\{0\} \\
v_{i}^{2}=1, & \forall i \in V \cup\{0\}
\end{array}
$$

where $v_{i} \in \mathbb{R}^{n+1}$. Constraints (2) are triangular inequalities, which must be satisfied by the vertex cover. In an 'integral' solution of (SDP) (which would correspond to a vertex cover of $G$ ), vertices that are picked coincide with $v_{0}$, while vertices that are not picked coincide with $-v_{0}$. In general though, an optimal solution of (SDP) will not be 'integral'.

In fact one can strengthen this SDP relaxation for VC by adding all the so called triangle inequalities:

$$
\begin{array}{rrrr}
\min \sum_{i=1}^{n} \frac{1+v_{0} v_{i}}{2} \text { s.t. } & \\
\left(v_{0}-v_{i}\right)\left(v_{0}-v_{j}\right)=0, & \forall(i, j) \in E \\
\left(v_{i}-v_{j}\right)\left(v_{i}-v_{k}\right) \geq 0, & \forall i, j, k \in V \cup\{0\} \\
\left(v_{i}+v_{j}\right)\left(v_{i}-v_{k}\right) \geq 0, & \forall i, j, k \in V \cup\{0\} \\
\left(v_{i}+v_{j}\right)\left(v_{i}+v_{k}\right) \geq 0, & \forall i, j, k \in V \cup\{0\} \\
v_{i}^{2}=1, & \forall i \in V \cup\{0\}
\end{array}
$$

This relaxation is in fact equivalent to the following relaxation: We add $n$ more 'shadow' points to (SDP) so that for every unit vector $v_{i}, i=1, \ldots, n$ we add unit vector $v_{i}^{\prime}$ which is the antipodal of $v_{i}$, i.e., $v_{i} v_{i}^{\prime}=-1, \forall i$. Let $V^{\prime}$ be the set of shadow points. Note that in an integral solution of (SDP), exactly half $(n)$ of the points in $V \cup V^{\prime}$ coincide with $v_{0}$ and the other half coincide with $-v_{0}$. Therefore the following must hold

$$
\sum_{i, j \in V \cup V^{\prime}}\left|v_{i}-v_{j}\right|^{2}=4 n^{2}
$$

where every pair $(i, j)$ appears only once in the sum. (Hence the set $V \cup V^{\prime}$ is $1 / 2$-spread in the terminology of [1]). In addition, the triangular inequalities (2) must also hold when we extend $V$ with $V^{\prime}$. Hence we have the following strengthened SDP: 


$$
\begin{array}{rlr}
\min \sum_{i=1}^{n} \frac{1+v_{0} v_{i}}{2} \text { s.t. } & \\
\left(v_{0}-v_{i}\right)\left(v_{0}-v_{j}\right) & =0, & \\
\left(v_{i}-v_{j}\right)\left(v_{i}-v_{k}\right) & \geq 0, & \forall(i, j) \in E \\
v_{i}^{2} & =1, & \forall i, j, k \in V \cup V^{\prime} \cup\{0\} \\
v_{i} v_{i}^{\prime} & =-1, & \\
\sum_{i, j \in V \cup V^{\prime}}\left|v_{i}-v_{j}\right|^{2} & =4 n^{2} &
\end{array}
$$

where $v_{i}, v_{i}^{\prime} \in \mathbb{R}^{d}$ for some $d \gg \log n$. Constraint (8) is in fact unnecessary since it is always satisfied by a set of points and their antipodals, but we include it in order to point out that this relaxation defines a spread metric as defined in [1]. Now we can use results of [1] to find an approximate VC.

For any $\varepsilon>0$, we define the following two sets of graph vertices:

$$
\begin{aligned}
& S_{1}:=\left\{v \in V: v_{0} v>\varepsilon\right\} \\
& S_{2}:=\left\{v \in V \cup V^{\prime}:-\varepsilon \leq v_{0} v \leq \varepsilon\right\}
\end{aligned}
$$

For now, we concentrate our attention on $S_{2}$. Note that in $S_{2}$ we have included also shadow points. In fact, note that if $v_{i} \in V$ belongs to $S_{2}$ then its shadow $v_{i}^{\prime} \in V^{\prime}$ belongs to $S_{2}$ as well, and vice-versa. In other words, $S_{2}$ contains both original points and their shadows.

\section{Lemma 1}

$$
\sum_{i, j \in S_{2}}\left|v_{i}-v_{j}\right|^{2}=4\left|S_{2}\right|^{2}
$$

Proof: Note that for a particular pair $i, j \in S_{2} \cap V$ we have $v_{i} v_{j}^{\prime}=v_{i}^{\prime} v_{j}=-v_{i} v_{j}$. So if we group the summation terms according to pairs of vertices $i, j \in S_{2} \cap V$, we get the lemma, due to cancellation of terms.

Let $\Delta, \sigma>0$ be two parameters to be determined later. Let $u$ be a random unit vector, and let

$$
\begin{aligned}
& S_{u}:=\left\{v \in S_{2}: u v \geq \frac{\sigma}{\sqrt{d}}\right\} \\
& T_{u}:=\left\{v \in S_{2}: u v \leq-\frac{\sigma}{\sqrt{d}} .\right\}
\end{aligned}
$$

Since $v_{i}=-v_{i}^{\prime}$, it is easy to prove the following

Lemma 2 If $v_{i} \in S_{u}$ for some $v_{i} \in V$, then $v_{i}^{\prime} \in T_{u}$, and vice-versa, if $v_{i}^{\prime} \in T_{u}$, then $v_{i} \in S_{u}$. The same holds with the roles of $S_{u}, T_{u}$ interchanged.

As a result of Lemma 2, $S_{u} \cup T_{u}$ contains only pairs of points in $V$ with their shadow points, and each such pair is separated between $S_{u}, T_{u}$, and $\left|S_{u}\right|=\left|T_{u}\right|$. Moreover, the following easy fact also holds:

\section{Lemma 3}

$$
\text { If } \left.\begin{array}{c}
v_{i} \in S_{u}, v_{j} \in T_{u}, \quad\left|v_{i}-v_{j}\right|^{2} \leq \Delta \\
v_{i} \in S_{u}, v_{j} \in T_{u},\left|v_{i}^{\prime}-v_{j}\right|^{2} \leq \Delta \\
v_{i}^{\prime} \in S_{u}, v_{j}^{\prime} \in v_{u}^{\prime},\left|v_{i}^{\prime}-v_{j}^{\prime}\right|^{2} \leq \Delta
\end{array}\right\} \Longrightarrow\left\{\begin{array}{c}
v_{j}^{\prime} \in S_{u}, v_{i}^{\prime} \in T_{u},\left|v_{i}^{\prime}-v_{j}^{\prime}\right|^{2} \leq \Delta \\
v_{j}^{\prime} \in S_{u}, v_{i} \in T_{u},\left|v_{i}-v_{j}^{\prime}\right|^{2} \leq \Delta \\
v_{j} \in S_{u}, v_{i}^{\prime} \in T_{u},\left|v_{i}^{\prime}-v_{j}\right|^{2} \leq \Delta \\
v_{j} \in S_{u}, v_{i} \in T_{u},\left|v_{i}-v_{j}\right|^{2} \leq \Delta
\end{array}\right.
$$


Let $c^{\prime}>0$ be another parameter which will be defined later. We modify the procedure SET-FIND of [1] as follows:

- If $\left|S_{u}\right|<2 c^{\prime}\left|S_{2}\right|$ or $\left|T_{u}\right|<2 c^{\prime}\left|S_{2}\right|$ then we HALT (just like in [1]).

- Otherwise, pick any $x \in S_{u}, y \in T_{u}$ such that $|x-y|^{2} \leq \Delta$. Then, because of Lemma 3, the corresponding pair of antipodal points $y^{\prime} \in S_{u}, x^{\prime} \in T_{u}$ also satisfy $\left|x^{\prime}-y^{\prime}\right|^{2} \leq \Delta$. Delete $x, x^{\prime}, y, y^{\prime}$. Repeat until no such $x, y$ can be found.

Note that initially $T_{u}$ contains the antipodal points of $S_{u}$ (Lemma 2), and every deletion eliminates two points from each of $S_{u}, T_{u}$, and these four actually form two (a point in $V$, its shadow point in $V^{\prime}$ ) pairs. Therefore, in the end, the remaining points in $S_{u}$ are exactly the antipodal points of $T_{u}$ (or both $S_{u}, T_{u}$ are empty). As in [1], $|x-y|^{2}>\Delta, \forall x \in S_{u}, y \in T_{u}$. One can define the parameters $c^{\prime}, \sigma$ so that, initially, $S_{u}, T_{u}$ are big with high probability:

Lemma 4 [Lemma 4 in [1]] For every positive $c<1 / 3$, there are $c^{\prime}, \sigma>0$ such that the probability (over the choice of $u$ ) is at least $c / 8$ that the initial sets $S_{u}, T_{u}$ defined above have size at least $2 c^{\prime}\left|S_{2}\right|$.

Proof: From Lemma 1 and application of Lemma 4 of [1].

In fact, since $S_{u}$ initially (and throughout the running of the algorithm) contains the antipodal points of $T_{u},\left|S_{u}\right|=\left|T_{u}\right|=\left|S_{2}\right| / 2$ before the algorithm starts running no matter which $u$ we choose, therefore $c^{\prime}=1 / 4$.

One of the main results of [1] is to show that, with high probability over $u$, not many points are deleted before SET-FIND terminates. Note that the points removed form a matching (at every step, $x$ is matched to $y$, and $x^{\prime}$ is matched to $\left.y^{\prime}\right)$. Theorem 5 in [1] shows that, with $\Delta=O\left(\log ^{-2 / 3} n\right)$, the probability that SET-FIND removes a matching of size $c^{\prime}\left|S_{2}\right|$ is $o(1)$. Hence the final $S_{u}, T_{u}$ of SET-FIND have size $\geq c^{\prime}\left|S_{2}\right|$ with probability $\Omega(1)$, and $\left|S_{u}\right|=\left|T_{u}\right|$. In what follows, we assume that $S_{u}, T_{u}$ are the big final sets we get with high probability from SET-FIND.

Lemma 5 If $\varepsilon \leq \Delta / 4$, then there is no edge $(i, j) \in E$ such that $v_{i}, v_{j} \in V$ belong both to $S_{u}$ or both to $T_{u}$.

Proof: W.l.o.g. suppose that there is $(i, j) \in E$ such that $v_{i}, v_{j} \in S_{u}$. Then their shadow (antipodal) points belong to $T_{u}$, i.e., $v_{i}^{\prime}, v_{j}^{\prime} \in T_{u}$. Since $v_{i}, v_{j} \in S_{2}$ and constraint (4) holds, we have that

$$
v_{i} v_{j}=v_{0} v_{i}+v_{0} v_{j}-1 \leq-(1-2 \varepsilon) .
$$

Since $v_{i}^{\prime} \in T_{u}$ and $v_{j} \in S_{u}$ are not deleted in SET-FIND, $\left|v_{i}^{\prime}-v_{j}\right|^{2}>\Delta$, or, equivalently, $\left|v_{i}+v_{j}\right|^{2}>\Delta$. This implies that

$$
v_{i} v_{j}>-1+\frac{\Delta}{2} \text {. }
$$

But (9) and (10) together imply that $\varepsilon>\Delta / 4$ which contradicts the hypothesis.

From now on we set $\varepsilon:=\Delta / 4>0$. Since $\left|S_{u}\right|=\left|T_{u}\right| \geq c^{\prime}\left|S_{2}\right|$, and the two sets contain antipodal points, one of them (w.l.o.g. let's assume that this is $S_{u}$ ), contains at least $\frac{c^{\prime}\left|S_{2}\right|}{2}$ points from $V$. Let $I$ be this set of points from $V$. Lemma 5 implies that $I$ is an independent set of $G$ of size at least $c_{0}\left|S_{2}\right|$, where $c_{0}:=c^{\prime} / 2>0$. We return the set $S:=S_{1} \cup\left(S_{2} \backslash\left(I \cup V^{\prime}\right)\right)$ as our vertex cover.

Lemma $6 S$ is a vertex cover of $G$. 
Proof: If there is $(i, j) \in E$ with $v_{i}, v_{j} \in V \backslash\left(S_{1} \cup S_{2}\right)$, we have (by the definition of $\left.S_{1}, S_{2}\right)$ that $v_{0} v_{i}<-\varepsilon$ and $v_{0} v_{j}<-\varepsilon$, which implies that $v_{0} v_{i}+v_{0} v_{j}-1<-1-2 \varepsilon$. Then constraint (4) implies that $v_{i} v_{j}<-1-2 \varepsilon$, a contradiction. Also, since $I$ is an independent set, not both of $v_{i}, v_{j}$ can belong to it. If $v_{i} \in I$ and $v_{j} \in V \backslash\left(S_{1} \cup S_{2}\right)$, then $v_{0} v_{i} \leq \varepsilon$ and $v_{0} v_{j}<-\varepsilon$, therefore constraint (4) implies that $v_{i} v_{j}<-1$, a contradiction. We conclude that every edge must have at least one of its endpoints in $S$.

Our main result is the following

Theorem $1|S| \leq\left(2-\Theta\left(\frac{1}{\log ^{2 / 3} n}\right)\right) V C(G)$.

Proof: We follow the analysis of Halperin [7]. From (SDP') and the definition of $S_{1}, S_{2}$ we have that

$$
V C(G) \geq\left|S_{1}\right| \frac{1+\varepsilon}{2}+\left|S_{2} \backslash V^{\prime}\right| \frac{1-\varepsilon}{2}
$$

or, equivalently,

$$
\left|S_{1}\right| \leq \frac{2 \cdot V C(G)}{1+\varepsilon}-\left|S_{2} \backslash V^{\prime}\right| \frac{1-\varepsilon}{1+\varepsilon}
$$

Hence

$$
|S|=\left|S_{1}\right|+\left|S_{2} \backslash V^{\prime}\right|-|I| \stackrel{(11)}{\leq} \frac{2}{1+\varepsilon} V C(G)+\left|S_{2} \backslash V^{\prime}\right|\left(\frac{2 \varepsilon}{1+\varepsilon}-c_{0}\right) .
$$

Note that for $\Delta=\Theta\left(\log ^{-2 / 3} n\right), \frac{2 \varepsilon}{1+\varepsilon}=\Theta\left(\log ^{-2 / 3} n\right)<c_{0}$, for big enough $n$. Therefore,

$$
|S| \leq \frac{2}{1+\varepsilon} V C(G)=\left(2-\Theta\left(\log ^{-2 / 3} n\right)\right) \cdot V C(G) .
$$

Very recently, J. Lee proved that the SET-FIND algorithm of [1] can also be used to obtain their stronger result [9], i.e., $\Delta$ can be as big as $\Theta(1 / \sqrt{\log n})$. Therefore we can get the following strengthening of Theorem 1:

Theorem $2|S| \leq\left(2-\Theta\left(\frac{1}{\sqrt{\log n}}\right)\right) V C(G)$.

Theorem 2 can be somewhat strengthened by noticing that in the proof of Theorem 1 we just need to pick $\Delta$ so that $\frac{2 \varepsilon}{1+\varepsilon}<c_{0}$, and therefore [1] and [9] imply that if $x:=1 / \Delta^{2}$, it is enough for $x$ to be the solution of equation

$$
\frac{x}{\log x}=c \log n
$$

where $c>0$ is a constant (cf. [4] for more details on solving this equation through Lambert's $W$ function).

\section{The weighted case}

The following is a semidefinite-programming relaxation of weighted Vertex Cover (VC) for a graph $G=(V, E)$ with $n$ nodes: 


$$
\begin{array}{rrr}
\min \sum_{i=1}^{n} w_{i} \cdot \frac{1+v_{0} v_{i}}{2} \text { s.t. } & \\
\left(v_{0}-v_{i}\right)\left(v_{0}-v_{j}\right) & =0, & \forall(i, j) \in E \\
\left(v_{i}-v_{j}\right)\left(v_{i}-v_{k}\right) & \geq 0, & \forall i, j, k \in V \cup\{0\} \\
v_{i}^{2}=1, & \forall i \in V \cup\{0\}
\end{array}
$$

where $w_{i}$ is the integral weight of node $i$. Let $W:=\sum_{i=1}^{n} w_{i}$.

In order to apply the methods of Section 2, we solve (SDP') with the weights incorporated in the objective function, and replace every $v_{i}$ by $w_{i}$ copies of $v_{i}\left(v_{i}^{\prime}\right.$ is also replaced by $w_{i}$ copies of $\left.v_{i}^{\prime}\right)$. In fact we don't need to do this replacement in practice, but this mental experiment is helpful in order to see how the unweighted case applies here, too. Note that this new set of vectors still satisfies the triangular inequalities, and Lemmata 4 through 6 in Section 2 apply here as well with $n:=W$. Note that SET-FIND can be made to run in polynomial time in this case (recall that we don't really do the replacement of $v_{i}$ with $w_{i}$, all we need to do is to keep track of how much weight remains for each node after each matching). Now Theorem 1 (and hence Theorem 2) can be proven in the same way as before, if we replace the cardinality of sets $|\cdot|$ with their weights $w(\cdot)$.

\section{Open problems}

Obviously one of the biggest open problems in theoretical computer science is the exact determination of the approximability of VC. There is a big gap between the hardness and the approximability results. Unfortunately, the SET-FIND procedure of [1] is limited to a gap of at most $\Theta(1 / \sqrt{\log n})$ by the embedding of the $\log n$-dimensional hypercube: in this case any two subsets of linear size are closer than $\Theta(1 / \sqrt{\log n})$ (this simple fact was pointed out to us by James R. Lee).

We couldn't extend our techniques to other problems related to VC, for example the maximum independent set problem (IS), and we don't know whether this is possible (Halperin's [7] techniques, on the contrary, can be applied to IS). Another extension of VC is the vertex cover problem in hypergraphs. We don't know how to extend our techniques to this problem as well. Therefore we leave the application of the results above to these and other problems as an open question.

Finally, we point out that we don't know what the integrality gap of the strengthened SDP relaxation (SDP') used above is. A weaker formulation, that doesn't contain all the triangle inequalities but is equivalent to Schrijver's $\theta^{\prime}$ function [6], was proven to have an integrality gap of $2-\varepsilon$ for any constant $\varepsilon>0$ by Charikar [3]. It would be interesting to show the same result for the stronger SDP.

Acknowledgements I am grateful to Sanjeev Arora for reading an earlier draft of this work and for bringing [9] to my attention.

\section{References}

[1] S. Arora, S. Rao, and U. Vazirani. Expander flows, geometric embeddings and graph partitioning. In Proc. of 36th STOC, pp. 222-231, 2004.

[2] R. Bar-Yehuda and S. Even. A local-ratio theorem for approximating the weighted vertex cover problem. Annals of Discrete Mathematics, 25, pp. 27-45, 1985. 
[3] M. Charikar. On semidefinite programming relaxations for graph coloring and vertex cover. In Proc. of 13th SODA, pp. 616-620, 2002.

[4] R. M. Corless, G. H. Gonnet, D. E. G. Hare, D. J. Jeffrey, and D. E. Knuth. On the Lambert W Function. Advances in Computational Mathematics, vol. 5, pp. 329-359, 1996.

[5] I. Dinur and S. Safra. On the importance of being biased (1.36 hardness of approximating VertexCover). Annals of Mathematics, to appear. Also in Proc. of 34th STOC, 2002.

[6] M. Goemans and J. Kleinberg. The Lovász Theta Function and a Semidefinite Programming Relaxation of Vertex Cover. SIAM Journal on Discrete Mathematics 11(2), pp. 196-204, 1998.

[7] E. Halperin. Improved approximation algorithms for the vertex cover problem in graphs and hypergraphs. SIAM J. on Computing, 31(5), pp. 1608-1623, 2002. Also in Proc. of 11th SODA, pp. 329-337, 2000.

[8] R. Karp. Reducibility among combinatorial problems. in R. E. Miller and J. W. Thatcher (eds.) Complexity of Computer Computations, Plenum Press, NY, pp. 85-103.

[9] J. R. Lee. On distance scales, embeddings, and efficient relaxations of the cut cone. SODA 2005 (to appear), see http://www.eecs.berkeley.edu/ j jrl.

[10] B. Monien and E. Speckenmeyer. Ramsey numbers and an approximation algorithm for the vertex cover problem. Acta Informatica, 22, pp. 115-123, 1985.

[11] C. E. Papadimitriou and M. Yannakakis. Optimization, approximation, and complexity classes. JCSS, 43(3), pp. 425-440, 1991. 